\title{
Synthesis and Characterization of Different Molecular Weights Polyacrylamide
}

\author{
Raid Saleh Shatat ${ }^{1 *}$, S. Kalimulla Niazi ${ }^{1}$,Azlan Ariffin ${ }^{2}$ \\ ${ }^{1}$ Riyadh Colleges of Dentistry and Pharmacy, 84891 Riyadh 11681, Kingdom of Saudi Arabia. Tel: 920000842. \\ Fax: 920000843 \\ ${ }^{2}$ School of Material and Mineral Resources, UniversitiSains Malaysia, KampusKejuruteraan, 14300 \\ NibongTebal, SeberangPerai Selatan, Penang, Malaysia \\ *corresponding Author
}

\begin{abstract}
Polyacrylamides of varying molecular weights (very low, low, medium, high and very high) were synthesized using free radical polymerization and characterized by Infra Red (IR) spectroscopy, viscosity measurements, glass transition temperature (Tg) measurement and thermal gravimetric analysis (TGA). Thioglycolic acid and hydrogen peroxide were used as initiators to synthesis very low and low molecular weight polyacrylamides respectively. Molecular weights (medium, high and very high) polyacrylamides were synthesized using potassium persulfate and $N, N, N^{\prime}, N^{\prime}$ - tetramethylethylenediaminesystem as initiators. The synthesis of medium, high and very high molecular weight polyacrylamides were achieved by increasing the concentration of acrylamide monomer. Fourier Transform Infrared Spectroscopy (FTIR) of polyacrylamides showed strong absorption at wave number $1655 \mathrm{~cm}^{-1}$ due to the presence of primary amide band. The results also showed that the increase in the intrinsic viscosity and glass transition temperature were proportional to the increase in molecular weight of polyacrylamide. On the other hand it can be seen an increase in molecular weight caused a decrease of weight loss \%.
\end{abstract}

Keywords:polyacrylamide, molecular weight, free radical polymerization, characterization.

\section{Introduction}

Natural polyelectrolytes (e.g. starch, gelatin, tannins, etc.) and inorganic electrolytes (e.g. aluminum sulphate, ferric sulphate, ferric chloride, etc.) have been traditionally as agents in water and wastewater treatment for the removal of colloidal suspended solids. The operations of these agents involve particle agglomeration by coagulation and flocculation processes (1). The uses of synthetic organic polyelectrolytes in water and wastewater treatment have been introduced in late 1950. Synthetic polyelectrolytes have been used extensively as coagulation aids or to replace inorganic electrolytes coagulants completely (2). The use of synthetic polyelectrolytes as flocculant is increasing rapidly. Polyelectrolytes increase floc strength allowing bigger and stronger flocs to be formed. The increased floc strength can improve settling characteristics. Synthetic organic polyelectrolytes are known to be more efficient than the inorganic electrolytes agents in the removal of suspended colloidal particles (3). The effectiveness of synthetic organic polyelectrolytes as flocculants increases with increasing molecular size, the limit of which is only dictated by problems in solubility. Polyelectrolytes having molar masses greater than 10 million $\mathrm{g} / \mathrm{mol}$ do not dissolve readily but tend to form gel lumps known as "fish eyes". Polymers of lower molar mass are highly soluble in water due to the hydration of functional groups along the molecule. Polyacrylamide shows high solubility in water, while it is insoluble in most other common organic liquids such as methanol, ethanol and acetone. However, despite this highly hydrophilic character, the main technical application of polyacrylamide is for flocculation of aqueous suspension and related purposes. Polyacrylamides can be classified to three categories: non-ionic, anionic and cationic polyacrylamides (4). Polyacrylamides were used extensively as a flocculant in the clarification of water in industrial and municipal processes (5). Cationic polyelectrolytes are a class of polyelectrolytes that derive their unique properties from the density and distribution of positive charges along a macromolecular backbone as well as molecular weight. Cationic functional groups can strongly interact with suspended, negatively charged particles or oil droplets and are useful for many applications including wastewater treatment and papermaking. There is a greater variety of molecular structures in the cationic family than any of the other polyelectrolytes. The present study involves the synthesis and characterization of different molecular weight polyacrylamides using free radical polymerization. 


\section{Chemicals}

\section{Materials and methods}

Acrylamide (Fluka, 99\%), potassium persulfate (98\%), and N,N,N',N'- tetramethylethylenediamine (TMEDA) (98\%)were purchased from Fluka, Germany as laboratory grade .Thioglycolic acid, hydrogen peroxide, acetone, ethanol and methanol were purchased from Merck, Germany as laboratory grade.

Equipment and instrumentation

The polymerization reaction was carried out in a Memmert water bath (thermostatically controlled, heating capacity up to $100^{\circ} \mathrm{C}$ ). A 1 litre five-necked Pyrex glass reactor was suitably placed into the Memmert water bath. The reactor was equipped with a mechanical stirrer, condenser equipped with water collector, thermometer and nitrogen gas (Oxygen Free Nitrogen) inlet tube.

\section{Recrystallization of chemicals}

Acrylamide (AM) was dissolved in acetone at $56^{\circ} \mathrm{C}$ (boiling point of acetone) to form a saturated solution, the solution was then filtered to remove insoluble particles and allowed to cool so that the dissolved substance is crystallized, the solid crystals were separated from acetone by filtration under suction using a Büchner funnel, washed with cold acetone and then dried in vacuum for $24 \mathrm{~h}$ at $60{ }^{\circ} \mathrm{C}$. This method was repeated twice. Potassium persulfate was recrystallized twice from distilled water at $100^{\circ} \mathrm{C}$ (boiling point of distilled water) using the same procedure described above. The rest of the chemicals were used without further purification.

\section{Synthesis of different molecular weight polyacrylamides}

Acrylamide monomer is crystalline. It is relatively stable and is soluble in water and many organic solvents (acetone, ethanol, methanol, etc.). Acrylamide polymerizes easily by conventional free radical methods. Solutions of acrylamide in water polymerize at $40{ }^{\circ} \mathrm{C}$ under the influence of nearly all free radical sources including persulfates, peroxides, azo compounds and redox couples. The synthesis of different molecular weight polyacrylamides is strongly influenced by temperature, solvent, type of initiator, concentration of the initiator and concentration of the monomer.

\section{Synthesis of very low molecular weight polyacrylamide}

Under nitrogen atmosphere, a mixture of acrylamide monomer $(10 \mathrm{gm})$, thioglycolic acid $(0.20 \mathrm{gm})$ and tetrahydrofuran (THF) (20ml) were placed in $500 \mathrm{ml}$ five-necked Pyrex glass reactor equipped with a reflux condenser and a mechanical stirrer placed in a constant temperature bath at $40^{\circ} \mathrm{C}$. The polymerization was performed for $2 \mathrm{~h}$ under stirring. The polyacrylamide was isolated and re-precipitated by pouring its solution into acetone. It was then dried under vacuum at $60^{\circ} \mathrm{C}$ for $24 \mathrm{~h}$.

\section{Synthesis of low molecular weight polyacrylamide}

The acrylamide monomer was polymerized in distilled water, using hydrogen peroxide as the initiator. Nitrogen gas was bubbled inside a five-necked Pyrex glass reactor containing the acrylamide monomer (20 gm), distilled water $(200 \mathrm{ml})$ as a solvent and hydrogen peroxide $(0.5 \mathrm{ml})$ as initiator. The reactor was placed in a constant temperature bath $90^{\circ} \mathrm{C}$ for $3 \mathrm{~h}$ under stirring. The polyacrylamide was isolated and re-precipitated by pouring its solution into acetone. It was then dried under vacuum at $60^{\circ} \mathrm{C}$ for $24 \mathrm{~h}$.

\section{Synthesis of medium, high and very high molecular weight polyacrylamide}

High purity of the monomer and initiators and the absence of oxygen are essential in the polymerization process. High concentration of monomers, low concentration of initiators are required to synthesized medium, high and very high molecular weight polyacrylamides. Different molecular weight (medium, high and very high) polyacrylamides were synthesized using the potassium persulfate and N,N, N', N'tetramethylethylenediamine (TMEDA) system as initiator. Increasing the concentration of acrylamide monomer will increase the molecular weight of the resulting polyacrylamide. Thus, under nitrogen atmosphere 15,30 and $50 \mathrm{gm}$ of acrylamide monomer were polymerized in $(500 \mathrm{ml})$ distilled water as a solvent and the $(0.135 \mathrm{gm})$ potassium persulfate/(180 $\mathrm{l})$ TEMDA system as initiator to produce medium, high and very high molecular weight polyacrylamides respectively.

The mixture were placed in $500 \mathrm{ml}$ five-necked Pyrex glass reactor equipped with a reflux condenser and mechanical stirrer placed in a constant temperature bath at $40^{\circ} \mathrm{C}$. The polymerization was performed for $2 \mathrm{~h}$ under stirring. The polyacrylamide was isolated and re-precipitated by pouring its solution into acetone. It was then dried under vacuum at $60^{\circ} \mathrm{C}$ for $24 \mathrm{~h}$. 


\section{Characterization of synthesized polyacrylamide}

The polymer characterization is almost as important as polymer preparation. Without characterization, polymer synthesis becomes additional exercise.

\section{Fourier Transform Infrared (FTIR) Spectra analysis}

Fourier transform infrared spectroscopy (FTIR) is a very important step in polymer characterization. FTIR is used very extensively for the characterization of polymer materials in polymer chemistry (6). FTIR technique is normally used in the investigation of polymer structure and analysis of their functional groups.

Nicolet FTIR machine, Model: Avator 360 FTIR spectrometer, manufactured by Nicolet analytical instrument USA Inc., USA was used in this study. Analysis was conducted by identification of acrylamide monomer and synthesized products (polyacrylamide).

Potassium bromide (KBr) pellet was prepared by using a sampling device. Approximately 5-10 mg of the sample powder and 90-95 $\mathrm{mg}$ of $\mathrm{KBr}$ was blended, triturated and mixed well with agate mortar and pestle for $5 \mathrm{~min}$. The mixture was transferred to the IR sampling device and then compacted using an IR hydraulic press for 60 seconds. $\mathrm{KBr}$ pellet was placed into FTIR window and then scanned by taking blank spectrum. After completion of the spectrum of the blank, $\mathrm{KBr}$ pellet was taken off from the window and $\mathrm{KBr}$ pellet thinly coated with test sample was placed again in the FTIR window to measure the spectrum of the sample. On average, 10 scans were used for the synthesized sample.

\section{Glass transition temperature $\left(T_{g}\right)$ measurement}

Glass transition temperature $\left(\mathrm{T}_{\mathrm{g}}\right)$ is an important characteristic property of a polymeric material because its fabrication and/or applications depend on it. It is a feature of polymeric materials that they may be entirely amorphous, partly amorphous and partly crystalline. The glass transition temperature $\left(\mathrm{T}_{\mathrm{g}}\right)$ of all synthesized polyacrylamides were measured using Perkin-Elmer, Differential Scanning Calorimetry (DSC) thermal analyzer. It is equipped with a DSC cell programmed at a heating rate of $10{ }^{\circ} \mathrm{C} / \mathrm{min}$., measuring temperature range was $25^{\circ} \mathrm{C}$ to $250^{\circ} \mathrm{C}$.Samples were prepared for DSC measurement by weighting between 5.0 $\mathrm{mg}$ to $10.0 \mathrm{mg}$ of dry polymer into an aluminum pan. The pan was then sealed with an aluminum cap. Similarly, an empty aluminum pan was used as reference.

\section{Viscosity average molecular weight of polyacrylamide}

The viscosity average molecular weight of polyacrylamides was carried out by determining the intrinsic viscosities of the polyacrylamides using Ubbelohede viscometer, no. 4809 ASTM at $25 \pm 0.1^{\circ} \mathrm{C}$. The polyacrylamide solutions were prepared in distilled water. The intrinsic viscosity calculations were made for polyacrylamide after filtration through 250-micron sieve to remove any undissolved 'gel' lumps.

Dilute solution viscosity method was used to determine the viscosity average molecular weight of the polyacrylamide samples by determining their intrinsic viscosities. Test sample was prepared by dissolving $0.5 \mathrm{~g}$ of polyacrylamide in $100 \mathrm{ml}$ distilled water at room temperature using magnetic stirrer. The resultant solution was filtered through 250-micron sieve to remove any undissolved 'gel' lumps. A series of polyacrylamide working solution with concentrations ranging between $5 \times 10^{-3}$ and $1 \mathrm{X}^{-3} \mathrm{~g} / \mathrm{ml}$ were prepared by appropriate dilution of the test sample with distilled water.

Approximate amounts of the solution were transferred into Ubbelhode viscometer and the viscometer placed inside a water bath set at $25 \pm 0.1^{\circ} \mathrm{C}$ and allowed to equilibrate. The efflux time (i.e. the time taken for the liquid inside the viscometer to pass from the upper meniscus to the lower meniscus was determined). An average of the three readings (which did not differ by more than 3 seconds) was taken for each sample.

\section{Thermal gravimetric analysis (TGA)}

Samples were prepared for TGA measurements by weighting between 5.0 to $10.0 \mathrm{gm}$ of dry polyacrylamide into aluminum pan. The pan was then sealed with aluminum cap.

Thermal gravimetric analysis (TGA) of different molecular weight polyacrylamides were carried out in the temperature range of $30-600^{\circ} \mathrm{C}$ at a heating rate of $10^{\circ} \mathrm{C} / \mathrm{min}$ under nitrogen using Perkin-Elmer.

\section{Results and Discussion}

The molecular weight of polyacrylamide can be varied by several techniques, including monomer concentration, initiator concentration and initiator type and polymerization temperature. The monomer concentration can be increased to $50 \%$ or more for high molecular weight polyacrylamide since the viscous polymer molecular weight is the internal phase. High purity of the monomer and initiators and the absence of oxygen are essential in the polymerization process.

The polymerization reaction at different temperaturesof acrylamide monomer with different initiators i.e. thioglycolicacid $\left(40^{\circ} \mathrm{C}\right)$, hydrogen peroxide $\left(90^{\circ} \mathrm{C}\right)$, and potassium persulfate/ N,N,N',N'tetramethylethylenediamine (TMEDA) $\left(40^{\circ} \mathrm{C}\right)$, gave the targeted very low, low, medium, high and very high 
molecular weight polyacrylamide products (shown in Table 1). The synthesis of high and very high molecular weight polyacrylamide was achieved by increasing the concentration of acrylamide monomer using potassium persulfate/TMEDA as initiatorat moderate temperature.

The synthesis route of the preparation of polyacrylamide is shown in table 1 . The choice of initiators and monomer concentrations were also studied.

Table 1: Type of initiator used and resultant molecular weight polyacrylamide

\begin{tabular}{|l|l|}
\hline Type of initiator used & Resultant molecular weight polyacrylamide \\
\hline Thioglycolicacid & Very low \\
\hline Hydrogen peroxide & Low \\
\hline Potassium persulfate/ (TMEDA) & Medium, high and very high \\
\hline
\end{tabular}

The structure of different molecular weight polyacrylamides was determined through the study of FTIR spectra. Glass transition temperature $\left(\mathrm{T}_{\mathrm{g}}\right)$, viscosity average molecular weight and thermal gravimetric analysis of polyacrylamide were also carried out.

\section{Synthesis of very low molecular weight polyacrylamide}

Very low molecular weight polyacrylamide was synthesized by free radical polymerization using thioglycolic acid as initiator at moderate temperature $\left(40^{\circ} \mathrm{C}\right)$. The ratio of thioglycolic acid to acrylamide monomer was about $0.02(\mathrm{~g} / \mathrm{g})$ and polyacrylamide with molecular weight of about $20,000 \mathrm{~g} / \mathrm{mole}$ was produced. The use of thioglycolic acid as a chain transfer agent has been shown to be a very effective method for the production of very low molecular weight polyacrylamide (7).

Thioglycolic acid is used as a chain transfer agent where it is desirable to decrease the molecular weight in radical polymerization. Thioglycolic acid enters the propagation scheme by giving up a proton to the growing radical chain, terminating it, but possibly initiating a new chain.

The thioglycolic acid was found to be an acceptable initiator for the production of a very low molecular weight polyacrylamide at moderate temperature and maximum yield was obtained without any unwanted side products. The product was a white coloured liquid with very low viscosity.

Acetone was used to isolate and re-precipitate the polyacrylamide product from its liquid solution and dried under vacuum at $60^{\circ} \mathrm{C}$ for $24 \mathrm{~h}$ to produce a yield of $70-75 \%$ of very low molecular weight polyacrylamide.

\section{Synthesis of low molecular weight polyacrylamide}

Low molecular weight polyacrylamide was synthesized using hydrogen peroxide as initiator and the reaction was carried out at high temperature $\left(90^{\circ} \mathrm{C}\right)$ to give polyacrylamide free of contaminates detected by FTIR spectroscopy. The product was a low viscosity white colour liquid. The polyacrylamide product was precipitated from its liquid solution using acetone and then dried under vacuum at $60^{\circ} \mathrm{C}$ for $24 \mathrm{~h}$ to produce a yield of $70-75 \%$ of low molecular weight polyacrylamide.

The molecular weight was found to be $170 \times 10^{3} \mathrm{~g} / \mathrm{mol}$ using viscosity measurements, which is considered to be low.

\section{Synthesis of medium, high and very high molecular weight polyacrylamide}

Potassium persulfate/TMEDA system as initiator was used for the synthesis of medium, high and very high molecular weight polyacrylamides. TMEDA exerts a high promoting effect on the free radical polymerization initiated by potassium persulfate. The rate of polymerization increases by about three times in the presence of TMEDA. Potassium persulfate/TMEDA system can reduce the reaction time and react at moderate temperature $\left(40^{\circ} \mathrm{C}\right)$.

Potassium persulfate/ TMEDA system was found to be an acceptable redox initiator for the production of medium, high and very high molecular weight polyacrylamides at moderate temperature and a maximum yield of 90-95\% was obtained without any unwanted side products. The product was a high viscosity white colour liquid. Polyacrylamide was precipitated from the solution using acetone and then dried under vacuum at $60^{\circ} \mathrm{C}$ for $24 \mathrm{~h}$. Figure 1 shows the effect of monomer concentration on molecular weight of polyacrylamide. As shown, the concentration of acrylamide monomer strongly affects the molecular weight of polyacrylamide. An increase in acrylamide monomer concentration caused an increase in molecular weights of polyacrylamide.

The fact that high concentration of monomer will initiate many sites, resulting in longer polyacrylamide chains, on the other hand shorter polyacrylamide chains at lower concentration of monomer. This is reflected in their corresponding intrinsic viscosity values.

Intrinsic viscosity of a polymer is a measure of its hydrodynamic volume in solution, which in turn depends upon its molecular weight, nature of the solvent and the temperature of the medium (8). For polymers of low molecular weight, the hydrodynamic volume will be low and thus it has low intrinsic viscosity value. 
Further, in the case of high molecular weight polymers the hydrodynamic volume will be high and therefore higher will be its intrinsic viscosity. This has been observed in practice (Figure 1).

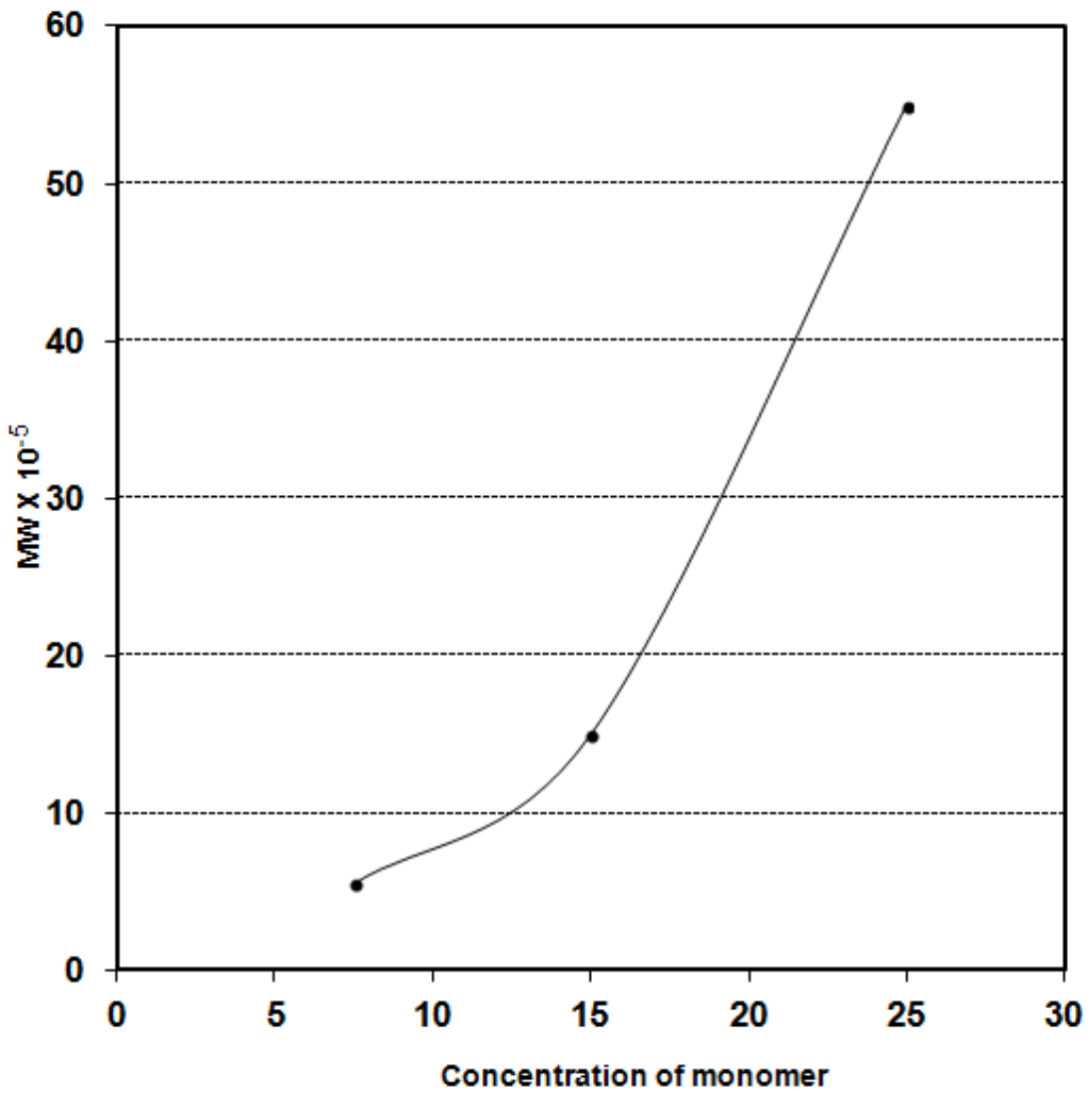

Figure 1: Influence of monomer concentration on molecular weight of polyacrylamide

\section{Characterization}

\section{Fourier Transform Infrared (FTIR) spectra analysis}

Fourier Transform Infrared Spectroscopy (FTIR) of polyacrylamide showed strong absorption at wave number $1655 \mathrm{~cm}^{-1}$ due to the presence of a primary amide band which is used to distinguish true polyacrylamide from the alternative polymerization product poly $(\beta$-alanine) which has instead a secondary amide band at 1555 $\mathrm{cm}^{-1}(4)$. The band for $(\mathrm{N}-\mathrm{H})$ stretching vibration appears at $3448 \mathrm{~cm}^{-1}$ of the $\mathrm{NH}_{2}$ group. Further, the absence of $(\mathrm{C}==\mathrm{C})$ band which usually appears at $1610 \mathrm{~cm}^{-1}$ proved that the acrylamide monomer has been completely converted to polyacrylamide and no residue of monomer is present in the polymer (9) studied the FTIR spectra of polyacrylamide, their finding is in a good agreement with our results.

\section{Glass transition temperature $\left(\mathbf{T}_{\mathrm{g}}\right)$ measurement}

The glass transition is a physical change from the glassy to the liquid state which occurs in amorphous solids when they are heated. This point is characterised by the glass transition temperature determined by Differential Scanning Calorimetry (DSC). The glass transition temperature, $\mathrm{T}_{\mathrm{g}}$, of very low, low, medium, high and very high molecular weight polyacrylamide appeared at 100.944, 109.077, $120.811,148.727$ and $154.461^{\circ} \mathrm{C}$ respectively.

This shows that up to these relatively high temperatures the polyacrylamide was in the glassy state and thus relatively resistant to the ingress (way in) or egress (way out) of small molecule penetrate, and it is necessary to use higher temperatures to put the polymer in the rubbery state where adsorbed solvent molecules are sufficiently mobile to be desorbed.Molecular weight influences $\mathrm{T}_{\mathrm{g}}$ significantly. At lower molecular weights where the excess of free volume associated with chain ends is significant. As molecular weight increases the concentration of chain ends decreases until its contribution to the free volume becomes negligible.

Louie and Hartwig observed an increase in glass transition temperature with increase the molecular weight of polymer. The results of this work are in a good agreement with their work (10). 


\section{Determination of approximate molecular weight}

Viscometry and application of Mark-Houwink equation is one of the simplest and most rapid methods used for determining the molecular weights of polymers. Molecular weight of the polyacrylamide samples can be estimated from the intrinsic viscosity values. Mark-Houwink equation is generally employed for the estimation of molecular weight of linear polymers.

The molecular weights of polyacrylamide samples were determined by determining their intrinsic viscosities using Ubbelohede viscometer. The polyacrylamide samples were filtered with 250-micron sieve after dissolution in distilled to prevent particulate impurities from entering the capillary of the viscometer and affect the flow rate of the solution through it.

The determination of viscosity average molecular weight from the measurement of intrinsic viscosity require working in a dilute solution using a suitable solvent. Therefore, in this study, the concentration of polyacrylamide solutions ranging from $1 \times 10^{-3}$ to $5 \times 10^{-3} \mathrm{~g} / \mathrm{ml}$ were prepared by appropriate dilution of the test sample with distilled water. By increasing the concentration of polyacrylamide, the intermolecular interactions increased leading to the formation of intermolecular complexes, resulting in the enhancement of densities. Therefore, relatively higher values in the efflux time at higher concentration of polyacrylamide solution were predictable.

The values of relative, specific, reduced and inherent viscosities of the various concentrations of the very low, low, medium, high and very high polyacrylamides were determined.

It was found that by increasing the polyacrylamide concentrations from $1 \times 10^{-3}$ to $5 \times 10^{-3} \mathrm{~g} / \mathrm{ml}$ the efflux time increased. As a result, the relative viscosity increased with an increase in the polyacrylamide concentration. Other viscosities namely specific and reduced viscosities also increased with an increase in the polyacrylamide concentration. On the other hand, the inherent viscosity was inversely proportional to the increase in polyacrylamide concentration.

The intrinsic viscosity $[\eta]$, which is defined as the intercept on the abscissa at zero concentration, was determined by plotting the reduced viscosity of the solution of different concentrations of polyacrylamide solutions. Intrinsic viscosity of the very low, low, medium, high and very high polyacrylamides samples were obtained.The results of intrinsic viscosity and viscosity average molecular weight are tabulated in table 2 . It can be seen that the increase in the intrinsic viscosity was proportional to the increase in molecular weight of polyacrylamide.

Table 2: Intrinsic viscosity and viscosity average molecular weight of different polyacrylamides

\begin{tabular}{|l|l|l|}
\hline Polyacrylamide & Intrinsic viscosity $(\mathrm{g} / \mathrm{dl})$ & Viscosity average molecular weight $(\mathrm{g} / \mathrm{mole})$ \\
\hline Very low & 13 & 19,040 \\
\hline Low & 76 & 173,089 \\
\hline Medium & 186 & 508,563 \\
\hline High & 456 & $1,625,402$ \\
\hline Very high & 1535 & Over 5 million \\
\hline
\end{tabular}

\section{Thermal gravimetric analysis (TGA)}

TGA is used primarily for determining thermal stability of polymers. TGA method is based on continuous measurement of weight on a sensitive balance as sample temperature is increased in an inert atmosphere. Data are recorded as a thermogram of weight versus temperature. Initially weight loss may arise from evaporation of residual moisture or solvent, but at higher temperatures it results from polymer decomposition (11). The thermal stability of the different molecular weight polyacrylamides has been studied by TGA from $50^{\circ} \mathrm{C}$ up to approximately $600^{\circ} \mathrm{C}$. The very high molecular weight polyacrylamide displayed the highest stability in the series, indicating the importance of the molecular weight for thermal stability. On the other hand, very low molecular weight polyacrylamide displayed the lowest stability.

The increase in molecular weight caused a decrease of weight loss $\%$. The residue at $600^{\circ} \mathrm{C}$ being $11.67 \%, 14.68 \%, 17.83 \%, 19.45 \%$, and $22.55 \%$ for the cases of very low, low, medium, high and very high molecular weight polyacrylamides respectively. The activation energy values for various stages of decomposition were calculated from thermal gravimetric analysis (TGA). The TGA of polyacrylamide was three-staged, as observed before by Ismail et al. (12). First, the loss of water which is nonstoichiometric occurred. This is followed by the subsequent loss of ammonia and other gaseous products from the polyacrylonitrile structure formed during the decomposition of polyacrylamide and partly from the remaining polyacrylamide in the course of heating to $600^{\circ} \mathrm{C}$.

For polyacrylamide moisture release takes place at a slow rate over the temperature zone of $40-200^{\circ} \mathrm{C}$ accounting for successive release of absorbed moisture and bound (H-bounded) water. A relatively high mass loss zone then follows till about $400^{\circ} \mathrm{C}$ which correspond to cyclization of polyacrylamide and associated liberation of $\mathrm{NH}_{3}$. A much sharper mass loss zone appears over the temperature zone of $400-500^{\circ} \mathrm{C}$ accounting 
for large-scale thermal degradation with the liberation of $\mathrm{H}_{2}, \mathrm{CO}$ and $\mathrm{NH}_{3}$, leaving a residue of about $22 \%$ at $600^{\circ} \mathrm{C}(9)$.

\section{References}

[1]. Gill, R. I. S. (1985). The flocculation of kaolinite suspensions using cationic polyacrylamide. Ph.D, thesis, The University of Reading, Reading, Berkshire. England.

[2]. Culp, R.L. (1977). Direct filtration. J. Amer. Water Works Assoc., 69 (7), 378

[3]. Gill, R. I. S. and Herrington, T. M. (1988). Floc size studies on kaolin suspensions flocculated with cationic polyacrylamides, Colloid and surfaces, 32, 331-344.

[4]. Molyneux, P. (1982). Water-soluble synthetic polymers: properties and behavior. Volume I. Florida: CRC Press.

[5]. Ives, K.J. (1978). The Scientific Basis of Flocculation, NATO ASI series, Sijthoff and Noordhoof, Alphen aan den Rijin, (Ed.).

[6]. Thompson, H. W. and Torkington, P. (1945). Trans. Farad. Soc., 41, 246.

[7]. $\quad \ddot{z}, \mathrm{~N}$ and Akar, A. (2000). Low molecular weight polyacrylic acid with pendant aminomethylenephosphonic acid groups. J. App. Poly. Sci., 78, 870-874.

[8]. Nayak, B. R. and Singh, R. P. (2001). Synthesis and characterization of grafted hydroxypropyl guar gum by ceric ion induced initiation. Eur. Poly. J., 37(8), 1655-1666

[9]. Ghosh, P. Chakrabarti, A. and Siddhanta S. K. (1999). Studies on stable aqueous polyaniline prepared with the use of polyacrylamide as the water soluble support polymer. Euro. Poly. J., 35(5), 803-813.

[10]. Louie, J. and Hartwig, J.F. (1997). Discrete high molecular weight triarylaminedendrimers prepared by palladium-catalyzed amination. J. Am. Chem. Sco., 119, 11659-11696.

[11]. Stevens, M. P. (1990). Polymer Chemistry an Introduction ( $2^{\text {nd }}$ ed.) 171-172.

[12]. Ismail, M. Vangani, V. Rakshit, A. K. (1997). Terpolymerization of acrylamide, acrylic acid, and acrylonitrile: synthesis and properties. J. App. Poly. Sci.,69, 217-228. 